\title{
A Crosstalk Between Brain Cholesterol Oxidation and Glucose Metabolism in Alzheimer's Disease
}

\author{
Paola Gamba*t, Erica Staurenghit, Gabriella Testa, Serena Giannelli, Barbara Sottero \\ and Gabriella Leonarduzzi
}

Department of Clinical and Biological Sciences, University of Turin, San Luigi Hospital, Turin, Italy

OPEN ACCESS

Edited by:

Eugenio Barone,

Sapienza University of Rome, Italy

Reviewed by:

Gérard Lizard,

Université de Bourgogne, France

Marzia Perluigi,

Sapienza University of Rome, Italy

*Correspondence:

Paola Gamba

paola.gamba@unito.it

${ }^{\dagger}$ These authors have contributed equally to this work

Specialty section: This article was submitted to

Neurodegeneration,

a section of the journal

Frontiers in Neuroscience

Received: 05 April 2019

Accepted: 14 May 2019

Published: 31 May 2019

Citation:

Gamba P, Staurenghi E, Testa G,

Giannelli S, Sottero B and

Leonarduzzi G (2019) A Crosstalk Between Brain Cholesterol Oxidation and Glucose Metabolism

in Alzheimer's Disease.

Front. Neurosci. 13:556.

doi: 10.3389/fnins.2019.00556
In Alzheimer's disease (AD), both cholesterol and glucose dysmetabolism precede the onset of memory deficit and contribute to the disease's progression. It is indeed now believed that oxidized cholesterol in the form of oxysterols and altered glucose uptake are the main triggers in $A D$ affecting production and clearance of $A \beta$, and tau phosphorylation. However, only a few studies highlight the relationship between them, suggesting the importance of further extensive studies on this topic. Recently, a molecular link was demonstrated between cholesterol oxidative metabolism and glucose uptake in the brain. In particular, 27-hydroxycholesterol, a key linker between hypercholesterolemia and the increased $A D$ risk, is considered a biomarker for reduced glucose metabolism. In fact, its excess increases the activity of the renin-angiotensin system in the brain, thus reducing insulin-mediated glucose uptake, which has a major impact on brain functioning. Despite this important evidence regarding the role of 27 hydroxycholesterol in regulating glucose uptake by neurons, the involvement of other cholesterol oxidation products that have been clearly demonstrated to be key players in $A D$ cannot be ruled out. This review highlights the current understanding of the potential role of cholesterol and glucose dysmetabolism in AD progression, and the bidirectional crosstalk between these two phenomena.

Keywords: Alzheimer's disease, cholesterol metabolism, oxysterols, glucose metabolism, insulin resistance, renin-angiotensin system

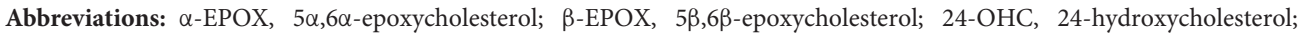

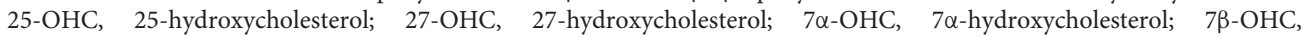
7 $\beta$-hydroxycholesterol; 7Hoca, $7 \alpha$-hydroxy-3-oxo-4-cholestenoic acid; 7-KC, 7-ketocholesterol; $\mathrm{A} \beta$, amyloid $\beta$; $\mathrm{ABC}$, ATP-binding cassette; ACE, angiotensin I-converting enzyme; Acetyl-CoA, acetyl coenzyme A; AD, Alzheimer's disease; Ang, angiotensin; AP, aminopeptidase; ApoE, apolipoprotein E; BBB, blood-brain barrier; BVR-A, biliverdin reductase A; $\mathrm{CH} 25 \mathrm{H}$, cholesterol 25-hydroxylase; CSF, cerebrospinal fluid; CYP27A1, cholesterol 27-hydroxylase; CYP46A1, cholesterol 24-hydroxylase; CYP7A1, cholesterol 7 $\alpha$-hydroxylase; CYP7B1, oxysterol $7 \alpha$-hydroxylase; GLUT, glucose transporter;

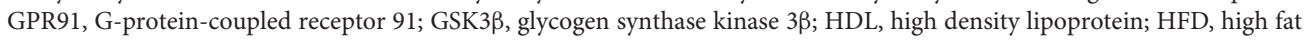
diet; HMG-CoA, 3-hydroxy-3-methylglutaryl-coenzyme A; HO-1, heme oxygenase 1; HSD3B7, 33-hydroxy-C27-steroid dehydrogenase/isomerase; IR, insulin resistance; IRAP, insulin-regulated aminopeptidase; IRS, insulin receptor substrate; LDLR, low density lipoprotein receptor; LRP, LDL receptor-like protein; LXR, liver X receptor; MAPK, mitogen-activated protein kinase; NFT, neurofibrillary tangle; PI3K, phosphoinositide 3-kinase; PPAR $\gamma$, peroxisome proliferator-activated receptor $\gamma$; RAS, renin-angiotensin system; SREBP, sterol-regulatory element-binding protein. 


\section{INTRODUCTION}

Several events in the brain contribute to $\mathrm{AD}$ development, including neuroinflammation, oxidative stress, $\mathrm{A} \beta$ toxicity, NFT formation, mitochondrial dysfunction, defective insulin signaling, decreased glucose utilization, and dysregulated cholesterol homeostasis. Deficiency in insulin signaling and IR, together with alteration in glucose and cholesterol metabolism, may lead to the occurrence of neuronal dysfunction and death and, consequently, to dementia. However, the molecular mechanisms involved in $\mathrm{AD}$ development are not completely clear, especially as regards the interaction between the different aspects of this pathology.

Cholesterol is particularly important in the brain since it is a major component of cell membranes, thus altered cholesterol metabolism may contribute to $\mathrm{AD}$ development (Gamba et al., 2015). Insulin is another important regulator of brain function. It affects neuronal synaptic function and plasticity, and glucose/cholesterol metabolism in the brain (Najem et al., 2014). Substantial glucose is required during memory processing especially in the hippocampus (McNay et al., 2001) and several neurodegenerative diseases are characterized by glucose hypometabolism (Teune et al., 2010). During AD progression, glucose dysmetabolism precedes the onset of memory deficit and it is speculated to predict the disease progression (Nordberg et al., 2010).

Both brain cholesterol and glucose dysmetabolism are recognized as important features of $\mathrm{AD}$, affecting the production and clearance of $\mathrm{A} \beta$ and tau phosphorylation, and inducing neurodegeneration (Sato and Morishita, 2015). Recently, a connection between these two processes has been highlighted; however, a more integrated understanding of the interactions between cholesterol and glucose metabolism is required in order to develop new therapeutic strategies to counteract AD. This review provides a brief summary of the rationale on the bidirectional relationship between two main risk factors in $\mathrm{AD}$ pathogenesis, i.e., brain cholesterol and glucose dysmetabolism due to insulin signaling deficiency.

\section{THE COMPLEX ROLE OF CHOLESTEROL IN THE BRAIN}

\section{Brain Cholesterol Metabolism}

The brain is the most cholesterol-rich organ, since it contains a quarter of the whole body non-esterified cholesterol pool (Dietschy, 2009). Cholesterol, as the main lipid component of neuronal and glial membranes and key constituent of myelin, plays essential roles in plasma membrane compartmentalization, signaling, myelination, and formation and maintenance of synapses (Petrov et al., 2017; Hussain et al., 2019).

Plasma and brain cholesterol pools are separated by two barriers: (i) the $\mathrm{BBB}$, that prevents lipoprotein-bound cholesterol uptake from the circulation; (ii) the bloodCSF barrier, through which plasma is ultrafiltered to form part of the CSF. In addition, CSF interfaces the brain interstitial fluid exchanging water, ions, and other molecules
(Johanson et al., 2011). Consequently, brain cholesterol metabolism is independent from that of peripheral tissues, and neurons rely on de novo-synthesized cholesterol delivery from astrocytes.

As shown in Figure 1, cholesterol is synthesized from Acetyl-CoA through reactions catalyzed by over 20 enzymes, including HMG-CoA reductase. Newly synthesized cholesterol is loaded into lipoproteins similar to HDLs, containing the ApoE. Lipidation and secretion of ApoE are mediated by $A B C$ transporters, such as ABCA1 and ABCG1. Then, lipoproteins are transported to neurons, where they are taken up by LDLRs and LRPs. Following receptor-mediated endocytosis, ApoE is recycled to the plasma membrane and cholesterol is used for cell membrane turnover and repair, myelin formation, synaptogenesis, and neurotransmitter release (Gamba et al., 2015; Petrov et al., 2016; Liao et al., 2017). In order to maintain brain cholesterol homeostasis, excess cholesterol is converted into oxysterols, important metabolites deriving from cholesterol enzymatic oxidation or auto-oxidation. Cholesterol is mainly converted into 24-OHC by CYP46A1, a cytochrome P-450 enzyme expressed by neurons. 24-OHC flows from the brain into the circulation across the $\mathrm{BBB}(\sim 99 \%)$ driven by the concentration gradient and, then, it is excreted by the liver in the form of bile acids (Björkhem et al., 2018; Dosch et al., 2019); less than $1 \%$ of $24-\mathrm{OHC}$ flows into the CSF (Lütjohann et al., 1996). Brain cholesterol is also oxidized into 27-OHC by the enzyme CYP27A1, expressed by neurons and glial cells. In contrast to $24-\mathrm{OHC}$, most of the cerebral 27-OHC derives from the peripheral circulation since CYP27A1 is expressed in most of the organs and tissues (Marwarha and Ghribi, 2015). 27$\mathrm{OHC}$ is indeed one of the major oxysterols in human circulation and its flux into the brain is likely driven by the concentration gradient, maintained by the high rate of its brain metabolism into 7Hoca by CYP7B1 and HSD3B7; subsequently, 7Hoca is eliminated in the systemic circulation and in the CSF (Meaney et al., 2007; Saeed et al., 2014; Björkhem et al., 2018). Both 24$\mathrm{OHC}$ and 27-OHC can, in turn, regulate cholesterol synthesis and transport from glia to neurons by acting on the nuclear LXR, that regulates the expression and synthesis of ApoE and ABCA1/ABCG1 (Czuba et al., 2017). In addition to 24-OHC and 27-OHC, other oxysterols are present in the brain (Testa et al., 2016). Besides enzymatic oxidation, cholesterol auto-oxidation can be induced by different compounds, such as lipid peroxides, free radical species, and metal cations, resulting in the formation of various oxysterols. Among them, $7 \alpha-\mathrm{OHC}, 7 \beta-\mathrm{OHC}, 7-\mathrm{KC}$, $25-\mathrm{OHC}, \alpha$-EPOX, and $\beta$-EPOX are the most representative. Both $7 \alpha-\mathrm{OHC}$ and $25-\mathrm{OHC}$ can also derive from cholesterol enzymatic oxidation, respectively by CYP7A1 and CH25H (Leoni and Caccia, 2013). These oxysterols flow from the brain into the systemic circulation and vice versa, crossing the BBB (Figure 1).

\section{The Involvement of Oxysterols in Alzheimer's Disease}

There has been growing evidence about the involvement of altered cholesterol metabolism in AD (Wood et al., 2014; Zarrouk et al., 2014, 2018; Gamba et al., 2015; Testa et al., 2018a). 


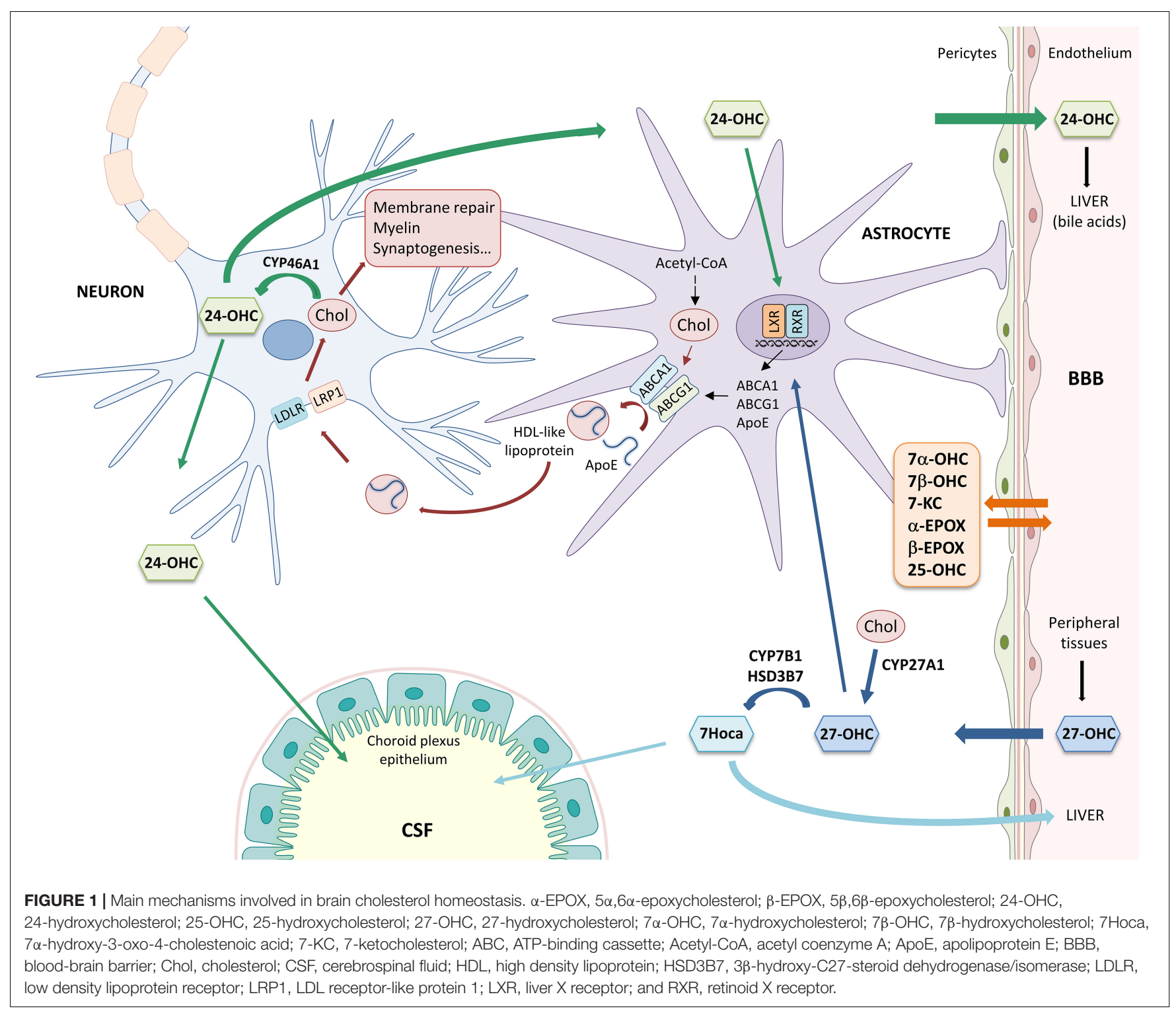

The AD brain, in particular the cortex and the hippocampus, is characterized by synaptic dysfunction, extracellular deposits of $\mathrm{A} \beta$ as senile plaques, and intracellular inclusions consisting of hyperphosphorylated tau protein as NFTs, all factors contributing to neuronal loss (Querfurth and LaFerla, 2010).

The presence of oxysterols in the brain could be one of the factors contributing to $\mathrm{AD}$ progression. It has been shown that some oxysterols (e.g., 27-OHC, 7 $\beta-\mathrm{OHC}$, and 7-KC) significantly increase in $\mathrm{AD}$ brains compared to healthy brains; in contrast, 24-OHC brain levels decrease likely due to neuronal loss (Hascalovici et al., 2009; Testa et al., 2016).

Concerning 27-OHC, its increased flux into the brain can be favored by hypercholesterolemia that induces oxidative stress, thus altering BBB permeability (Heverin et al., 2004; Dias et al., 2014). Moreover, under oxidative stress and inflammatory conditions, brain cholesterol metabolism into 27-OHC increases because the enzyme CYP27A1 is highly expressed by glial cells. Both these mechanisms cause the increase of $27-\mathrm{OHC} / 24-\mathrm{OHC}$ brain ratio (Marwarha and Ghribi, 2015). 27-OHC has been observed to promote pro-inflammatory molecule release (Testa et al., 2014), to increase A $\beta$ levels (Prasanthi et al., 2009; Gamba et al., 2014), in human neuroblastoma cell lines and both $\mathrm{A} \beta$ and hyperphosphorylated tau levels in rabbit organotypic hippocampal slices (Marwarha et al., 2010). Moreover, 27OHC has been recently demonstrated to impact on lysosomal membrane permeabilization and pyroptosis in co-cultured SH-SY5Y and C6 cells (Chen et al., 2019). In addition, increased $\mathrm{A} \beta$ plaques were found in the hippocampus of 27-OHC-treated mice (Zhang et al., 2018), and 27-OHC has been shown to induce synaptic dysfunction and to impair neuron morphology (Merino-Serrais et al., 2019).

As regards 24-OHC, contrasting effects have been reported: on the one hand it promotes neuroinflammation, $A \beta$ peptide 
production, oxidative stress, and cell death in neuronal cell lines (Gamba et al., 2011, 2014; Yamanaka et al., 2011; Testa et al., 2014); on the other hand, it has been reported to play an important role in regulating brain cholesterol metabolism via LXR, and to exert beneficial effects such as preventing tau hyperphosphorylation in SK-N-BE cells, suppressing $\mathrm{A} \beta$ production in SH-SY5Y cells, and regulating synaptic function in rat hippocampal neurons and slices (Paul et al., 2013; Urano et al., 2013; Testa et al., 2018b). These opposite effects may depend on 24-OHC concentration, since low concentrations $(1-10 \mu \mathrm{M})$ seem to induce adaptive responses and beneficial effects in neuronal cell lines as discussed by Testa et al. (2018a).

\section{THE INTERPLAY BETWEEN CHOLESTEROL AND GLUCOSE METABOLISM IN THE BRAIN}

\section{The Role of Oxysterols in Brain Insulin Resistance}

Insulin is an important regulator of brain cell function and metabolism: it affects neuronal synaptic function and plasticity and regulates both glucose and cholesterol metabolism. Like in peripheral tissues, insulin signaling in the brain is mediated by the binding of insulin to its receptor. Consequently, insulin receptor auto-phosphorylation leads to the phosphorylation of the IRS family, of which IRS1 is the best characterized. IRS1 activates two important signaling pathways: the PI3K/Akt pathway and the MAPK cascade (Akter et al., 2011). The activation of the insulin signaling cascade leads to the translocation of the insulin-sensitive GLUT4 to the plasma membrane to favor glucose uptake during memory-related cognitive functions (McEwen and Reagan, 2004).

However, the insulin-mediated glucose uptake in the brain is not as significant as in the periphery. Indeed, brain glucose uptake is also regulated by the cerebral RAS, which is essential for several brain functions, such as learning, memory, emotional responses, and processing of sensory information. A significant reduction of RAS activity has been reported in the AD brains (Mateos et al., 2008, 2011a,b). The downstream peptide Ang IV binds to its receptor, known as IRAP, which is localized in specialized vesicles containing GLUT4 within hippocampal neurons, as well as throughout other brain regions. This binding inhibits IRAP activity, thus preventing the cleavage of memory-enhancing peptides, and activates GLUT4 favoring glucose uptake, thus preserving cognitive functions (Wright and Harding, 2008).

Recently, a molecular link was demonstrated among cholesterol metabolism, brain glucose uptake, and the brain RAS, all of which are affected in neurodegenerative diseases. Besides being a link between hypercholesterolemia and the increased $\mathrm{AD}$ risk, 27-OHC is considered a biomarker for the reduced brain glucose metabolism in $\mathrm{AD}$ since it is able to increase brain RAS activity, thus impairing neuronal glucose uptake (Figure 2). In particular, 27-OHC is involved in the reduction of glucose uptake in the brain by modulating the activity of IRAP and GLUT4. To do this, 27-OHC increases the expression of two main factors involved in the cerebral RAS: AP-A, which transforms Ang II into Ang III, and AP-N, which degrades Ang IV (Ismail et al., 2017). Since Ang III and Ang IV have opposite effects (Ang III inhibits GLUT4 and activates IRAP and, vice versa, Ang IV activates GLUT4 and inhibits IRAP), it can be assumed that 27-OHC excess in the brain, as in the case of $\mathrm{AD}$, may reduce brain glucose uptake which has a major impact on brain functioning (Figure 2). In this connection, in vivo experiments demonstrated that intracerebroventricular injection of $10 \mu \mathrm{M} 27-\mathrm{OHC}$ in WT mice significantly reduces the levels of GLUT4 and increases the levels of AP-A, AP-N and IRAP in the hippocampus. Moreover, a decrease in GLUT4 levels and an enhancement in IRAP levels were observed in cortical and hippocampal primary neurons treated with $1 \mu \mathrm{M}$ 27-OHC (Ismail et al., 2017). The activation of IRAP by 27-OHC causes the cleavage of neuropeptides and thus contributes to memory deterioration (Lew et al., 2003). These results are supported by the fact that CYP27A1 overexpressing mice show decreased glucose metabolism and memory deficit (Ismail et al., 2017). In vitro experiments also demonstrated that treatments of rat primary neurons, astrocytes, and human neuroblastoma cells with 1-10 $\mu \mathrm{M}$ 27-OHC stimulate the production of angiotensinogen, the precursor of Ang I. Moreover, in AD the activity of ACE correlates with 27-OHC levels both in plasma and CSF (Mateos et al., 2011a), although ACE levels have been shown to be reduced in the CSF (Miners et al., 2009).

Several effects exerted by $27-\mathrm{OHC}$ on brain RAS have been observed to be mediated by $\mathrm{LXR} \beta$, since $27-\mathrm{OHC}$ is a good LXR ligand (Ismail et al., 2017). However, besides 27-OHC, other oxysterols have been identified as endogenous ligands for LXR, including 24S-OHC (Nagy et al., 2012). In fact, both $24 \mathrm{~S}-\mathrm{OHC}$ and $27-\mathrm{OHC}$ regulate the brain RAS in primary neurons and astrocytes through a LXR-dependent mechanism, by upregulating angiotensinogen, ACE and Ang II type 1 receptors, all involved in neuronal plasticity, learning, and memory (Mateos et al., 2011b). In addition to $\operatorname{LXR} \beta$, also $\operatorname{LXR} \alpha$ regulates glucose uptake since the LXR $\alpha$ binding site has been found in the GLUT4 promoter (Dalen et al., 2003).

In addition, the expression of GLUT4, together with the expression of other genes involved in glucose metabolism control, may be regulated by PPAR $\gamma$ (Komers and Vrána, 1998). This nuclear receptor is also involved in the increase of LRP1, a member of the LDL receptor family involved in cholesterol metabolism but also in AD pathogenesis (Shinohara et al., 2017). LRP1 participates in A $\beta$ uptake and metabolism, and in amyloid precursor protein trafficking (Xue-Shan et al., 2016). Moreover, LRP1 is strongly associated to IR because it is involved in the insulin receptor trafficking and intracellular signaling, as well as in glucose uptake in several tissues, but mainly in the brain (Actis Dato and Chiabrando, 2018). In this regard, neuronal LRP1 deficiency leads to a reduced insulin receptor localization in the plasma membrane, an impaired insulin signaling, and decreased glucose uptake due to the lack of GLUT3 and GLUT4 (Liu C.C. et al., 2015). 


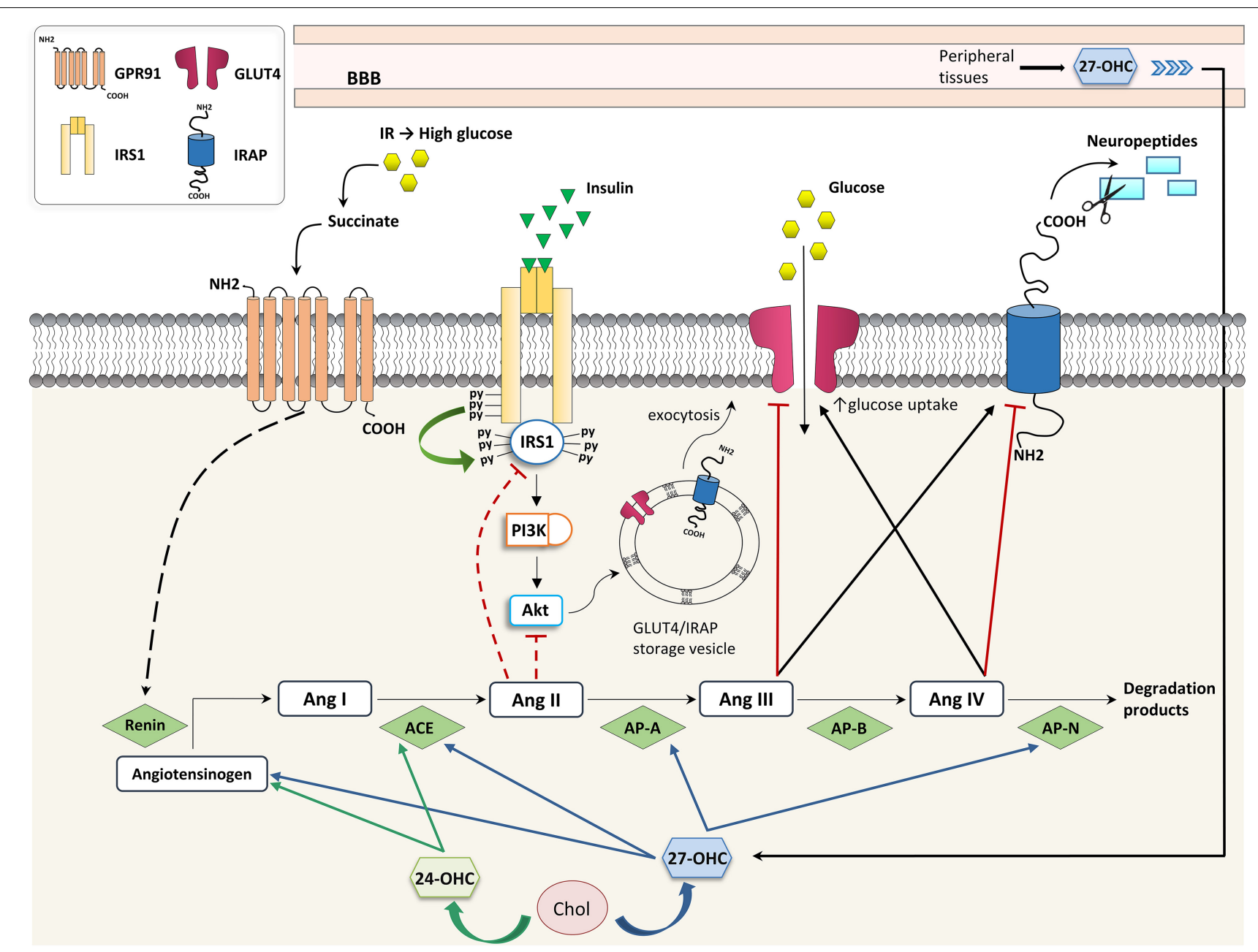

FIGURE 2 | Effects of oxysterols on RAS- and insulin-dependent glucose uptake. 24-OHC, 24-hydroxycholesterol; 27-OHC, 27-hydroxycholesterol; ACE, angiotensin I-converting enzyme; Ang, angiotensin; AP, aminopeptidase; BBB, blood-brain barrier; Chol, cholesterol; GLUT, glucose transporter; GPR91,

G-protein-coupled receptor 91; IR, insulin resistance; IRAP, insulin-regulated aminopeptidase; IRS, insulin receptor substrate; and PI3K, phosphoinositide 3-kinase.

Both the nuclear receptors PPAR $\gamma$ and LXRs are also implicated in the regulation of lipid metabolism. In this context, CYP27A1 gene expression, regulated by PPAR $\gamma$ and LXR signaling, results in increased levels of 27-OHC, which in turn up-regulates PPAR $\gamma$ and LXR-dependent processes (Szanto et al., 2004; An et al., 2017). Moreover, an oxysterol mixture compatible with that detectable in human hypercholesterolemic plasma, but not unoxidized cholesterol, has been shown to upregulate PPAR $\gamma$ (Leonarduzzi et al., 2010). In addition, oxidized derivatives of fatty acids, such as 9- and 13-hydroxyoctadecadienoic acid, both oxidized LDL components, activate PPAR $\gamma$ in macrophages (Nagy et al., 2012). Furthermore, macrophage-specific PPAR $\gamma$ knockout mice easily develop diet-induced obesity, glucose intolerance and IR (Hevener et al., 2007; Odegaard et al., 2007).

Brain IR is defined as the inadequate response to insulin by target cells and it has been considered a key feature in $\mathrm{AD}$ development since it is highly related to tau pathology. IR is, indeed, associated with higher tau levels in the CSF (Starks et al., 2015), and CSF tau predicts changes in brain glucose metabolism
(Dowling et al., 2015). It has been observed that in the AD brain there are lower levels of insulin and of insulin receptors, resulting in reduced PI3K/Akt signaling (Schubert et al., 2003, 2004) and GSK3 $\beta$ activation, responsible for NFT formation (Doble and Woodgett, 2003).

A direct crosstalk between high glucose levels induced by IR and RAS has been highlighted in kidneys. In particular, hyperglycemia induced by IR modulates RAS by leading to renin release through the binding of succinate to its receptor GPR91 (Peti-Peterdi et al., 2008); vice versa, RAS contributes to IR because Ang II impairs insulin signaling through IRS1 or PI3K/Akt inhibition, as shown in Figure 2 (Andreozzi et al., 2004). At present there is no evidence that this regulatory network exists also in the brain, but it has been demonstrated that 27-OHC and 24-OHC interfere in the brain's insulin-dependent glucose uptake through RAS.

The role of the HO-1/BVR-A system in the occurrence of IR in the brain, in particular in $\mathrm{AD}$, is gaining attention (Barone and Butterfield, 2015). 
The enzyme HO-1 is markedly overexpressed in cortical and hippocampal neurons and astroglia, and colocalizes with senile plaques and NFTs (Schipper et al., 1995). The upregulation of $\mathrm{HO}-1$, in particular by the astrocytic compartment, may confer cytoprotection by enhancing the break-down of prooxidant heme to the radical scavenging biliverdin and bilirubin. However, under certain conditions, heme-derived iron and $\mathrm{CO}$ may exacerbate intracellular oxidative stress by provoking free radical generation within mitochondria and other subcellular organelles. The interplay between brain HO-1 and cholesterol homeostasis may have important implications in the pathogenesis of $\mathrm{AD}$. In this connection, it has been demonstrated that HO-1 levels increase in the $\mathrm{AD}$ brain in parallel with the increased levels of oxysterols; indeed, HO-1 overexpression suppresses total cholesterol levels by favoring LXR-mediated cholesterol efflux, and enhances oxysterol formation (Vaya and Schipper, 2007; Hascalovici et al., 2014).

Brain IR may be due to increased phosphorylation of IRS1 on specific residues. In this connection, BVR-A is the kinase that phosphorylates and inhibits IRS1, consequently inhibiting the insulin signaling. For this reason, BVR-A is considered a novel mediator of IR. Interestingly, oxidative stress affects BVRA function resulting in the impairment of the insulin signaling in AD subjects (Barone et al., 2011, 2016).

\section{The Impact of High Fat Diet-Induced Hypercholesterolemia on Brain Insulin Resistance}

As one of the most cholesterol-rich organs, brain cholesterol homeostasis is tightly regulated; however, there is growing evidence that the brain lipid profile may be modified by HFDinduced hypercholesterolemia (Czuba et al., 2017).

In this connection, in $\mathrm{AD}$ and aging animal models it has been observed that HFD induces cognitive decline (Pancani et al., 2013; Knight et al., 2014). Long-term exposure to HFD results in the increase of plasma cholesterol and, most importantly, disturbs brain cholesterol homeostasis leading to $\mathrm{A} \beta$ accumulation, hyperphosphorylation of tau, and neuronal death (Vance, 2006). Moreover, the HFD triggers astrocytic activation in the murine hippocampi and increases the expression of proteins involved in cholesterol transport across brain cell membranes, such as ApoE, thus HFD has a great impact on brain cholesterol homeostasis (Chen et al., 2016). Reactive astrocytes release various inflammatory mediators, that can promote senile plaque and NFT formation that, in turn, contribute to the redox imbalance and inflammation. Cholesterol fed rabbits exhibit high levels of both reactive oxygen species and antioxidant enzyme HO-1 in the brain, and the increment of HO-1 correlates well with oxysterol levels (Hascalovici et al., 2014). It has also been shown that the brain levels of 27-OHC, transported from the systemic circulation, increased in high cholesterol fed rabbits, thus leading to neurodegeneration in the hippocampus (Brooks et al., 2017).

Besides the increased risk of $\mathrm{AD}$ induced by HFD because of brain cholesterol dysmetabolism (Stapleton et al., 2008), it has also been demonstrated that HFD induces hepatic IR and impairment of synaptic plasticity (Liu Z. et al., 2015). Additionally, in vivo studies demonstrated that HFD-induced peripheral IR and apoE\&4 gene variant synergistically impair cerebral insulin signaling (Zhao et al., 2017). The influence of HFD on the development of brain IR has been demonstrated by the presence, in the hippocampi of HFD fed mice, of elevated levels of phospho-IRS1 (Ser616) (Arnold et al., 2014), phosphoAkt (Ser473), and phospho-GSK3ß (Ser9) (Spinelli et al., 2017). Both short-term diet, with very high fat content, and longterm diet, with moderate fat, interfere with the insulin signaling pathways and induce IR in the brain (Arnold et al., 2014).

Furthermore, few studies highlight the importance of serum cholesterol in brain glucose uptake. Higher midlife serum total cholesterol levels are associated, in humans, with lower metabolic glucose rate in brain areas affected by $\mathrm{AD}$, such as precuneus, parietotemporal, and prefrontal regions, but also in frontal regions that are commonly affected by normal aging (Reiman et al., 2010). Moreover, high levels of blood cholesterol enhance RAS activity in the brain: high cholesterol fed mice show increased levels of the precursor angiotensinogen and of ACE (Mateos et al., 2011b). Moreover, HFD fed mice exhibit increased IRAP catalytic activity in the brain (Ismail et al., 2017).

\section{Insulin Resistance Regulates Cholesterol Metabolism in the Brain}

The crosstalk between cholesterol dysmetabolism and IR is bidirectional: not only hypercholesterolemia and altered cholesterol homeostasis affect IR, but also IR may, conversely, affect cholesterol metabolism; in fact, insulin can activate the transcription factors SREBPs involved in cholesterol biosynthesis (Suzuki et al., 2010). In addition, insulin increases cholesterol biosynthesis in SH-SY5Y and N2a cells, by upregulating 24dehydrocholesterol reductase, and HMG-CoA reductase through SREBP2, whereas A $\beta$-induced IR leads to dysregulation of cholesterol homeostasis (Najem et al., 2016). Moreover, insulindeficient diabetes leads to a reduced cholesterol synthesis in the brain due to lower expression of SREBP2 and of its downstream genes in the hypothalamus and in other brain regions, resulting in altered synaptic formation, and function (Suzuki et al., 2010, 2013). Conversely, cholesterol depletion in GT1-7 hypothalamic neuron-derived cells contributes to IR, alters autophagy, and enhances apoptosis induced by cytotoxic stress (Fukui et al., 2015).

\section{CONCLUSION}

Disruption of cholesterol and glucose metabolism are key players in $\mathrm{AD}$ onset and progression, however, the crosstalk between these two phenomena is not yet clear. Despite the important evidence regarding the role of certain oxysterols in regulating glucose uptake by neurons, it would be crucial to deepen their role in modulating the insulin signaling pathway in the brain in order to develop new strategies aimed at preventing or delaying $\mathrm{AD}$ development. 


\section{AUTHOR CONTRIBUTIONS}

All authors listed have made a substantial, direct and intellectual contribution to the work, and approved it for publication.

\section{REFERENCES}

Actis Dato, V., and Chiabrando, G. A. (2018). The role of low-density lipoprotein receptor-related protein 1 in lipid metabolism, glucose homeostasis and inflammation. Int. J. Mol. Sci. 19:E1780. doi: 10.3390/ijms19061780

Akter, K., Lanza, E. A., Martin, S. A., Myronyuk, N., Rua, M., and Raffa, R. B. (2011). Diabetes mellitus and Alzheimer's disease: shared pathology and treatment? Br. J. Clin. Pharmacol. 71, 365-376. doi: 10.1111/j.1365-2125.2010. 03830.x

An, Y., Zhang, D. D., Yu, H. L., Ma, W. W., Lu, Y. H., Liu, Q. R., et al. (2017). 27-Hydroxycholesterol regulates cholesterol synthesis and transport in C6 glioma cells. Neurotoxicology 59, 88-97. doi: 10.1016/j.neuro.2017. 02.001

Andreozzi, F., Laratta, E., Sciacqua, A., Perticone, F., and Sesti, G. (2004). Angiotensin II impairs the insulin signaling pathway promoting production of nitric oxide by inducing phosphorylation of insulin receptor substrate-1 on Ser312 and Ser616 in human umbilical vein endothelial cells. Circ. Res. 94, 1211-1218. doi: 10.1161/01.RES.0000126501.34994.96

Arnold, S. E., Lucki, I., Brookshire, B. R., Carlson, G. C., Browne, C. A., Kazi, H., et al. (2014). High fat diet produces brain insulin resistance, synaptodendritic abnormalities and altered behavior in mice. Neurobiol. Dis. 67, 79-87. doi: 10.1016/j.nbd.2014.03.011

Barone, E., and Butterfield, D. A. (2015). Insulin resistance in Alzheimer disease: is heme oxygenase-1 an Achille's heel? Neurobiol. Dis. 84, 69-77. doi: 10.1016/j. nbd.2015.02.013

Barone, E., Di Domenico, F., Cenini, G., Sultana, R., Cini, C., Preziosi, P., et al. (2011). Biliverdin reductase-a protein levels and activity in the brains of subjects with Alzheimer disease and mild cognitive impairment. Biochim. Biophys. Acta 1812, 480-487. doi: 10.1016/j.bbadis.2011.01.005

Barone, E., Di Domenico, F., Cassano, T., Arena, A., Tramutola, A., Lavecchia, M. A., et al. (2016). Impairment of biliverdin reductase-A promotes brain insulin resistance in Alzheimer disease: a new paradigm. Free Radic. Biol. Med. 91, 127-142. doi: 10.1016/j.freeradbiomed.2015.12.012

Björkhem, I., Leoni, V., and Svenningsson, P. (2018). On the fluxes of side-chain oxidized oxysterols across blood-brain and blood-CSF barriers and origin of these steroids in CSF. J. Steroid Biochem. Mol. Biol. 188, 86-89. doi: 10.1016/j. jsbmb.2018.12.009

Brooks, S. W., Dykes, A. C., and Schreurs, B. G. (2017). A high-cholesterol diet increases 27-hydroxycholesterol and modifies estrogen receptor expression and neurodegeneration in rabbit hippocampus. J. Alzheimers Dis. 56, 185-196. doi: 10.3233/JAD- 160725

Chen, Y. L., Wang, L. M., Chen, Y., Gao, J. Y., Marshall, C., Cai, Z. Y., et al. (2016). Changes in astrocyte functional markers and $\beta$-amyloid metabolismrelated proteins in the early stages of hypercholesterolemia. Neuroscience 316, 178-191. doi: 10.1016/j.neuroscience.2015.12.039

Chen, S., Zhou, C., Yu, H., Tao, L., An, Y., Zhang, X., et al. (2019). $27-$ hydroxycholesterol contributes to lysosomal membrane permeabilizationmediated pyroptosis in co-cultured SH-SY5Y cells and C6 cells. Front. Mol. Neurosci. 12:14. doi: 10.3389/fnmol.2019.00014

Czuba, E., Steliga, A., Lietzau, G., and Kowiański, P. (2017). Cholesterol as a modifying agent of the neurovascular unit structure and function under physiological and pathological conditions. Metab. Brain Dis. 32, 935-948. doi: 10.1007/s11011-017-0015-3

Dalen, K. T., Ulven, S. M., Bamberg, K., Gustafsson, J. A., and Nebb, H. I. (2003). Expression of the insulin-responsive glucose transporter GLUT4 in adipocytes is dependent on liver X receptor alpha. J. Biol. Chem. 278, 48283-48291. doi: 10.1074/jbc.M302287200

Dias, I. H., Polidori, M. C., and Griffiths, H. R. (2014). Hypercholesterolaemiainduced oxidative stress at the blood-brain barrier. Biochem. Soc. Trans. 42, 1001-1005. doi: 10.1042/BST20140164

\section{FUNDING}

This work was supported by the San Paolo Foundation (Turin) and the University of Turin (Italy).

Dietschy, J. M. (2009). Central nervous system: cholesterol turnover, brain development and neurodegeneration. Biol. Chem. 390, 287-293. doi: 10.1515/ BC. 2009.035

Doble, B. W., and Woodgett, J. R. (2003). GSK-3: tricks of the trade for a multi-tasking kinase. J. Cell Sci. 116, 1175-1186. doi: 10.1242/jcs.00384

Dosch, A. R., Imagawa, D. K., and Jutric, Z. (2019). Bile metabolism and lithogenesis: an update. Surg. Clin. North Am. 99, 215-229. doi: 10.1016/j.suc. 2018.12.003

Dowling, N. M., Johnson, S. C., Gleason, C. E., Jagust, W. J., and Alzheimer's Disease Neuroimaging Initiative (2015). The mediational effects of FDG hypometabolism on the association between cerebrospinal fluid biomarkers and neurocognitive function. Neuroimage 105, 357-368. doi: 10.1016/j.neuroimage. 2014.10.050

Fukui, K., Ferris, H. A., and Kahn, C. R. (2015). Effect of cholesterol reduction on receptor signaling in neurons. J. Biol. Chem. 290, 26383-26392. doi: 10.1074/ jbc.M115.664367

Gamba, P., Leonarduzzi, G., Tamagno, E., Guglielmotto, M., Testa, G., Sottero, B., et al. (2011). Interaction between 24-hydroxycholesterol, oxidative stress, and amyloid- $\beta$ in amplifying neuronal damage in Alzheimer's disease: three partners in crime. Aging Cell 10, 403-417. doi: 10.1111/j.1474-9726.2011.00681.x

Gamba, P., Guglielmotto, M., Testa, G., Monteleone, D., Zerbinati, C., Gargiulo, S., et al. (2014). Up-regulation of $\beta$-amyloidogenesis in neuron-like human cells by both 24- and 27-hydroxycholesterol: protective effect of $\mathrm{N}$-acetyl-cysteine. Aging Cell 13, 561-572. doi: 10.1111/acel.12206

Gamba, P., Testa, G., Gargiulo, S., Staurenghi, E., Poli, G., and Leonarduzzi, G. (2015). Oxidized cholesterol as the driving force behind the development of Alzheimer's disease. Front. Aging Neurosci. 7:119. doi: 10.3389/fnagi.2015. 00119

Hascalovici, J. R., Vaya, J., Khatib, S., Holcroft, C. A., Zukor, H., Song, W., et al. (2009). Brain sterol dysregulation in sporadic AD and MCI: relationship to heme oxygenase-1. J. Neurochem. 110, 1241-1253. doi: 10.1111/j.1471-4159. 2009.06213.x

Hascalovici, J. R., Song, W., Liberman, A., Vaya, J., Khatib, S., Holcroft, C., et al. (2014). Neural HO-1/sterol interactions in vivo: implications for Alzheimer's disease. Neuroscience 280, 40-49. doi: 10.1016/j.neuroscience.2014.09.001

Hevener, A. L., Olefsky, J. M., Reichart, D., Nguyen, M. T., Bandyopadyhay, G., Leung, H. Y., et al. (2007). Macrophage PPAR gamma is required for normal skeletal muscle and hepatic insulin sensitivity and full antidiabetic effects of thiazolidinediones. J. Clin. Invest. 117, 1658-1669. doi: 10.1172/JCI31561

Heverin, M., Bogdanovic, N., Lütjohann, D., Bayer, T., Pikuleva, I., Bretillon, L., et al. (2004). Changes in the levels of cerebral and extracerebral sterols in the brain of patients with Alzheimer's disease. J. Lipid Res. 45, 186-193. doi: 10.1194/jlr.M300320-JLR200

Hussain, G., Wang, J., Rasul, A., Anwar, H., Imran, A., Qasim, M., et al. (2019). Role of cholesterol and sphingolipids in brain development and neurological diseases. Lipids Health Dis. 18:26. doi: 10.1186/s12944-019-0965-z

Ismail, M. A., Mateos, L., Maioli, S., Merino-Serrais, P., Ali, Z., Lodeiro, M., et al. (2017). 27-Hydroxycholesterol impairs neuronal glucose uptake through an IRAP/GLUT4 system dysregulation. J. Exp. Med. 214, 699-717. doi: 10.1084/ jem.20160534

Johanson, C. E., Stopa, E. G., and McMillan, P. N. (2011). The blood-cerebrospinal fluid barrier: structure and functional significance. Methods Mol. Biol. 686, 101-131. doi: 10.1007/978-1-60761-938-3_4

Knight, E. M., Martins, I. V., Gümüsgöz, S., Allan, S. M., and Lawrence, C. B. (2014). High-fat diet-induced memory impairment in triple-transgenic Alzheimer's disease (3xTgAD) mice is independent of changes in amyloid and tau pathology. Neurobiol. Aging 35, 1821-1832. doi: 10.1016/j.neurobiolaging. 2014.02.010

Komers, R., and Vrána, A. (1998). Thiazolidinediones-tools for the research of metabolic syndrome X. Physiol. Res. 47, 215-225. 
Leonarduzzi, G., Gargiulo, S., Gamba, P., Perrelli, M. G., Castellano, I., Sapino, A., et al. (2010). Molecular signaling operated by a diet-compatible mixture of oxysterols in up-regulating CD36 receptor in CD68 positive cells. Mol. Nutr. Food Res. 54, S31-S41. doi: 10.1002/mnfr.200900493

Leoni, V., and Caccia, C. (2013). Potential diagnostic applications of side chain oxysterols analysis in plasma and cerebrospinal fluid. Biochem. Pharmacol. 86, 26-36. doi: 10.1016/j.bcp.2013.03.015

Lew, R. A., Mustafa, T., Ye, S., McDowall, S. G., Chai, S. Y., and Albiston, A. L. (2003). Angiotensin AT4 ligands are potent, competitive inhibitors of insulin regulated aminopeptidase (IRAP). J. Neurochem. 86, 344-350. doi: 10.1046/j. 1471-4159.2003.01852.x

Liao, F., Yoon, H., and Kim, J. (2017). Apolipoprotein E metabolism and functions in brain and its role in Alzheimer's disease. Curr. Opin. Lipidol. 28, 60-67. doi: 10.1097/MOL.0000000000000383

Liu, C. C., Hu, J., Tsai, C. W., Yue, M., Melrose, H. L., Kanekiyo, T., et al. (2015). Neuronal LRP1 regulates glucose metabolism and insulin signaling in the brain. J. Neurosci. 35, 5851-5859. doi: 10.1523/JNEUROSCI.5180-14.2015

Liu, Z., Patil, I. Y., Jiang, T., Sancheti, H., Walsh, J. P., Stiles, B. L., et al. (2015). High-fat diet induces hepatic insulin resistance and impairment of synaptic plasticity. PLoS One 10:e0128274. doi: 10.1371/journal.pone.0128274

Lütjohann, D., Breuer, O., Ahlborg, G., Nennesmo, I., Sidén, A., Diczfalusy, U., et al. (1996). Cholesterol homeostasis in human brain: evidence for an agedependent flux of 24S-hydroxycholesterol from the brain into the circulation. Proc. Natl. Acad. Sci. U.S.A. 93, 9799-9804. doi: 10.1073/pnas.93.18.9799

Marwarha, G., and Ghribi, O. (2015). Does the oxysterol 27-hydroxycholesterol underlie Alzheimer's disease-Parkinson's disease overlap? Exp. Gerontol. 68, 13-18. doi: 10.1016/j.exger.2014.09.013

Marwarha, G., Dasari, B., Prasanthi, J. R., Schommer, J., and Ghribi, O. (2010). Leptin reduces the accumulation of Abeta and phosphorylated tau induced by 27 -hydroxycholesterol in rabbit organotypic slices. J. Alzheimers Dis. 19, 1007-1019. doi: 10.3233/JAD-2010-1298

Mateos, L., Akterin, S., Gil-Bea, F. J., Spulber, S., Rahman, A., Björkhem, I., et al. (2008). Activity-regulated cytoskeleton-associated protein in rodent brain is down-regulated by high fat diet in vivo and by 27-hydroxycholesterol in vitro. Brain Pathol. 19, 69-80. doi: 10.1111/j.1750-3639.2008. 00174. $\mathrm{x}$

Mateos, L., Ismail, M. A., Gil-Bea, F. J., Leoni, V., Winblad, B., Björkhem, I., et al. (2011a). Upregulation of brain renin angiotensin system by 27 hydroxycholesterol in Alzheimer's disease. J. Alzheimers Dis. 24, 669-679. doi: 10.3233/JAD-2011-101512

Mateos, L., Ismail, M. A., Gil-Bea, F. J., Schüle, R., Schöls, L., Heverin, M., et al. (2011b). Side chain-oxidized oxysterols regulate the brain renin-angiotensin system through a liver X receptor-dependent mechanism. J. Biol. Chem. 286, 25574-25585. doi: 10.1074/jbc.M111.236877

McEwen, B. S., and Reagan, L. P. (2004). Glucose transporter expression in the central nervous system: relationship to synaptic function. Eur. J. Pharmacol. 490, 13-24. doi: 10.1016/j.ejphar.2004.02.041

McNay, E. C., McCarty, R. C., and Gold, P. E. (2001). Fluctuations in brain glucose concentration during behavioral testing: dissociations between brain areas and between brain and blood. Neurobiol. Learn. Mem. 75, 325-337. doi: 10.1006/ nlme.2000.3976

Meaney, S., Heverin, M., Panzenboeck, U., Ekström, L., Axelsson, M., Andersson, U., et al. (2007). Novel route for elimination of brain oxysterols across the blood-brain barrier: conversion into 7alpha-hydroxy-3-oxo-4-cholestenoic acid. J. Lipid Res. 48, 944-951. doi: 10.1194/jlr.M600529-JLR200

Merino-Serrais, P., Loera-Valencia, R., Rodriguez-Rodriguez, P., ParradoFernandez, C., Ismail, M. A., Maioli, S., et al. (2019). 27-Hydroxycholesterol induces aberrant morphology and synaptic dysfunction in hippocampal neurons. Cereb. Cortex 29, 429-446. doi: 10.1093/cercor/bhy274

Miners, S., Ashby, E., Baig, S., Harrison, R., Tayler, H., Speedy, E., et al. (2009). Angiotensin-converting enzyme levels and activity in Alzheimer's disease: differences in brain and CSF ACE and association with ACE1 genotypes. Am. J. Transl. Res. 1, 163-177.

Nagy, L., Szanto, A., Szatmari, I., and Széles, L. (2012). Nuclear hormone receptors enable macrophages and dendritic cells to sense their lipid environment and shape their immune response. Physiol. Rev. 92, 739-789. doi: 10.1152/physrev. 00004.2011
Najem, D., Bamji-Mirza, M., Chang, N., Liu, Q. Y., and Zhang, W. (2014). Insulin resistance, neuroinflammation, and Alzheimer's disease. Rev. Neurosci. 25, 509-525. doi: 10.1515/revneuro-2013-0050

Najem, D., Bamji-Mirza, M., Yang, Z., and Zhang, W. (2016). A $\beta$-induced insulin resistance and the effects of insulin on the cholesterol synthesis pathway and $A \beta$ secretion in neural cells. Neurosci. Bull. 32, 227-238. doi: 10.1007/s12264-0160034-9

Nordberg, A., Rinne, J. O., Kadir, A., and Långström, B. (2010). The use of PET in Alzheimer disease. Nat. Rev. Neurol. 6, 78-87. doi: 10.1038/nrneurol.2009.217

Odegaard, J. I., Ricardo-Gonzalez, R. R., Goforth, M. H., Morel, C. R., Subramanian, V., Mukundan, L., et al. (2007). Macrophagespecific PPARgamma controls alternative activation and improves insulin resistance. Nature 447, 1116-1120. doi: 10.1038/nature0 5894

Pancani, T., Anderson, K. L., Brewer, L. D., Kadish, I., DeMoll, C., Landfield, P. W., et al. (2013). Effect of high-fat diet on metabolic indices, cognition, and neuronal physiology in aging F344 rats. Neurobiol. Aging 34, 1977-1987. doi: 10.1016/j.neurobiolaging.2013.02.019

Paul, S. M., Doherty, J. J., Robichaud, A. J., Belfort, G. M., Chow, B. Y., Hammond, R. S., et al. (2013). The major brain cholesterol metabolite 24(S)hydroxycholesterol is a potent allosteric modulator of N-methyl-D-aspartate receptors. J. Neurosci. 33, 17290-17300. doi: 10.1523/JNEUROSCI.2619-13. 2013

Peti-Peterdi, J., Kang, J. J., and Toma, I. (2008). Activation of the renal reninangiotensin system in diabetes-new concepts. Nephrol. Dial. Transplant. 23, 3047-3049. doi: 10.1093/ndt/gfn377

Petrov, A. M., Kasimov, M. R., and Zefirov, A. L. (2016). Brain cholesterol metabolism and its defects: linkage to neurodegenerative diseases and synaptic dysfunction. Acta Naturae 8, 58-73.

Petrov, A. M., Kasimov, M. R., and Zefirov, A. L. (2017). Cholesterol in the pathogenesis of Alzheimer's, Parkinson's diseases and autism: link to synaptic dysfunction. Acta Naturae 9, 26-37.

Prasanthi, J. R., Huls, A., Thomasson, S., Thompson, A., Schommer, E., and Ghribi, O. (2009). Differential effects of 24-hydroxycholesterol and 27hydroxycholesterol on beta-amyloid precursor protein levels and processing in human neuroblastoma SH-SY5Y cells. Mol. Neurodegener. 4:1. doi: 10.1186/ 1750-1326-4-1

Querfurth, H. W., and LaFerla, F. M. (2010). Alzheimer's disease. N. Engl. J. Med. 362, 329-344. doi: 10.1056/NEJMra0909142

Reiman, E. M., Chen, K., Langbaum, J. B., Lee, W., Reschke, C., Bandy, D., et al. (2010). Higher serum total cholesterol levels in late middle age are associated with glucose hypometabolism in brain regions affected by Alzheimer's disease and normal aging. Neuroimage 49, 169-176. doi: 10.1016/j.neuroimage.2009.07. 025

Saeed, A., Floris, F., Andersson, U., Pikuleva, I., Lövgren-Sandblom, A., Bjerke, M., et al. (2014). 7 $\alpha$-hydroxy-3-oxo-4-cholestenoic acid in cerebrospinal fluid reflects the integrity of the blood-brain barrier. J. Lipid Res. 55, 313-318. doi: 10.1194/jlr.P044982

Sato, N., and Morishita, R. (2015). The roles of lipid and glucose metabolism in modulation of $\beta$-amyloid, tau, and neurodegeneration in the pathogenesis of Alzheimer disease. Front. Aging Neurosci. 7:199. doi: 10.3389/fnagi.2015.00199

Schipper, H. M., Cissém, S., and Stopam, E. G. (1995). Expression of heme oxygenase-1 in the senescent and Alzheimer-diseased brain. Ann. Neurol. 37, 758-768. doi: 10.1002/ana.410370609

Schubert, M., Brazil, D. P., Burks, D. J., Kushner, J. A., Ye, J., Flint, C. L., et al. (2003). Insulin receptor substrate-2 deficiency impairs brain growth and promotes tau phosphorylation. J. Neurosci. 23, 7084-7092. doi: 10.1523/ JNEUROSCI.23-18-07084.2003

Schubert, M., Gautam, D., Surjo, D., Ueki, K., Baudler, S., Schubert, D., et al. (2004). Role for neuronal insulin resistance in neurodegenerative diseases. Proc. Natl. Acad. Sci. U.S.A. 101, 3100-3105. doi: 10.1073/pnas.0308724101

Shinohara, M., Tachibana, M., Kanekiyo, T., and Bu, G. (2017). Role of LRP1 in the pathogenesis of Alzheimer's disease: evidence from clinical and preclinical studies. J. Lipid Res. 58, 1267-1281. doi: 10.1194/jlr.R0 75796

Spinelli, M., Fusco, S., Mainardi, M., Scala, F., Natale, F., Lapenta, R., et al. (2017). Brain insulin resistance impairs hippocampal synaptic plasticity and memory 
by increasing GluA1 palmitoylation through FoxO3a. Nat. Commun. 8:2009. doi: 10.1038/s41467-017-02221-9

Stapleton, P. A., James, M. E., Goodwill, A. G., and Frisbee, J. C. (2008). Obesity and vascular dysfunction. Pathophysiology 15, 79-89. doi: 10.1016/j.pathophys. 2008.04.007

Starks, E. J., Patrick, O., Grady, J., Hoscheidt, S. M., Racine, A. M., Carlsson, C. M., et al. (2015). Insulin resistance is associated with higher cerebrospinal fluid tau levels in asymptomatic APOE\&4 carriers. J. Alzheimers Dis. 46, 525-533. doi: 10.3233/JAD- 150072

Suzuki, R., Lee, K., Jing, E., Biddinger, S. B., McDonald, J. G., Montine, T. J., et al. (2010). Diabetes and insulin in regulation of brain cholesterol metabolism. Cell Metab. 12, 567-579. doi: 10.1016/j.cmet.2010.11.006

Suzuki, R., Ferris, H. A., Chee, M. J., Maratos-Flier, E., and Kahn, C. R. (2013). Reduction of the cholesterol sensor SCAP in the brains of mice causes impaired synaptic transmission and altered cognitive function. PLoS Biol. 11:e1001532. doi: 10.1371/journal.pbio.1001532

Szanto, A., Benko, S., Szatmari, I., Balint, B. L., Furtos, I., Rühl, R., et al. (2004). Transcriptional regulation of human CYP27 integrates retinoid, peroxisome proliferator-activated receptor, and liver $\mathrm{X}$ receptor signaling in macrophages. Mol. Cell. Biol. 24, 8154-8166. doi: 10.1128/MCB.24.18.8154-8166.2004

Testa, G., Gamba, P., Badilli, U., Gargiulo, S., Maina, M., Guina, T., et al. (2014). Loading into nanoparticles improves quercetin's efficacy in preventing neuroinflammation induced by oxysterols. PLoS One 9:e96795. doi: 10.1371/ journal.pone.0096795

Testa, G., Staurenghi, E., Zerbinati, C., Gargiulo, S., Iuliano, L., Giaccone, G., et al. (2016). Changes in brain oxysterols at different stages of Alzheimer's disease: their involvement in neuroinflammation. Redox Biol. 10, 24-33. doi: 10.1016/j.redox.2016.09.001

Testa, G., Rossin, D., Poli, G., Biasi, F., and Leonarduzzi, G. (2018a). Implication of oxysterols in chronic inflammatory human diseases. Biochimie 153, 220-231. doi: 10.1016/j.biochi.2018.06.006

Testa, G., Staurenghi, E., Giannelli, S., Gargiulo, S., Guglielmotto, M., Tabaton, M., et al. (2018b). A silver lining for 24-hydroxycholesterol in Alzheimer's disease: the involvement of the neuroprotective enzyme sirtuin 1. Redox Biol. 17, 423-431. doi: 10.1016/j.redox.2018.05.009

Teune, L. K., Bartels, A. L., de Jong, B. M., Willemsen, A. T., Eshuis, S. A., de Vries, J. J., et al. (2010). Typical cerebral metabolic patterns in neurodegenerative brain diseases. Mov. Disord. 25, 2395-2404. doi: 10.1002/mds. 23291

Urano, Y., Ochiai, S., and Noguchi, N. (2013). Suppression of amyloid- $\beta$ production by 24 S-hydroxycholesterol via inhibition of intracellular amyloid precursor protein trafficking. FASEB J. 27, 4305-4315. doi: 10.1096/fj.13231456

Vance, J. E. (2006). Lipid imbalance in the neurological disorder. Niemann-Pick C disease. FEBS Lett. 580, 5518-5524. doi: 10.1016/j.febslet.2006.06.008
Vaya, J., and Schipper, H. M. (2007). Oxysterols, cholesterol homeostasis, and Alzheimer disease. J. Neurochem. 102, 1727-1737. doi: 10.1111/j.1471-4159. 2007.04689.x

Wood, W. G., Li, L., Müller, W. E., and Eckert, G. P. (2014). Cholesterol as a causative factor in Alzheimer's disease: a debatable hypothesis. J. Neurochem. 129, 559-572. doi: 10.1111/jnc.12637

Wright, J. W., and Harding, J. W. (2008). The angiotensin AT4 receptor subtype as a target for the treatment of memory dysfunction associated with Alzheimer's disease. J. Renin Angiotensin Aldosterone Syst. 9, 226-237. doi: 10.1177/ 1470320308099084

Yamanaka, K., Saito, Y., Yamamori, T., Urano, Y., and Noguchi, N. (2011). 24(S)hydroxycholesterol induces neuronal cell death through necroptosis, a form of programmed necrosis. J. Biol. Chem. 286, 24666-24673. doi: 10.1074/jbc.M111. 236273

Xue-Shan, Z., Juan, P., Qi, W., Zhong, R., Li-Hong, P., Zhi-Han, T., et al. (2016). Imbalanced cholesterol metabolism in Alzheimer's disease. Clin. Chim. Acta 456, 107-114. doi: 10.1016/j.cca.2016.02.024

Zarrouk, A., Vejux, A., Mackrill, J., O’Callaghan, Y., Hammami, M., O’Brien, $\mathrm{N}$., et al. (2014). Involvement of oxysterols in age-related diseases and ageing processes. Ageing Res. Rev. 18, 148-162. doi: 10.1016/j.arr.2014.0 9.006

Zarrouk, A., Debbabi, M., Bezine, M., Karym, E. M., Badreddine, A., Rouaud, O., et al. (2018). Lipid biomarkers in Alzheimer's disease. Curr. Alzheimer Res. 15, 303-312. doi: 10.2174/1567205014666170505101426

Zhang, X., Xi, Y., Yu, H., An, Y., Wang, Y., Tao, L., et al. (2018). 27hydroxycholesterol promotes $A \beta$ accumulation via altering $A \beta$ metabolism in mild cognitive impairment patients and APP/PS1 mice. Brain Pathol. [Epub ahead of print],

Zhao, N., Liu, C. C., Van Ingelgom, A. J., Martens, Y. A., Linares, C., Knight, J. A., et al. (2017). Apolipoprotein E4 impairs neuronal insulin signaling by trapping insulin receptor in the endosomes. Neuron 96:115-129.e5. doi: 10 . 1016/j.neuron.2017.09.003

Conflict of Interest Statement: The authors declare that the research was conducted in the absence of any commercial or financial relationships that could be construed as a potential conflict of interest.

Copyright (C) 2019 Gamba, Staurenghi, Testa, Giannelli, Sottero and Leonarduzzi. This is an open-access article distributed under the terms of the Creative Commons Attribution License (CC BY). The use, distribution or reproduction in other forums is permitted, provided the original author(s) and the copyright owner(s) are credited and that the original publication in this journal is cited, in accordance with accepted academic practice. No use, distribution or reproduction is permitted which does not comply with these terms. 